\title{
Parallel Robots with Homokinetic Joints: The Zero-Torsion Case
}

\author{
Yuanqing $\mathrm{Wu}^{1}$, J.M. Selig ${ }^{2}$, and Marco Carricato ${ }^{1}$ \\ 1 University of Bologna, Bologna 40136, Italy, \\ yuanqing. wu@unibo.it, marco.carricato@unibo.it \\ 2 London South Bank University, London, UK, \\ seligjm@lsbu.ac.uk
}

\begin{abstract}
A two degree-of-freedom (DOF) homokinetic joint provides the freedom of spatially pointing directions without spinning (zero torsion). In this paper, we investigate structural synthesis of several classes of zero-torsion parallel robots using homokinetic joints.
\end{abstract}

Keywords: homokinetic joint, parallel robot, type synthesis, symmetric subspace

\section{Introduction}

The spherical joint (denoted $\mathcal{S}$ ) is a common machine element in the synthesis and design of parallel robots and machines (PMs) [1,2]. As shown in Fig. 1(a), the workspace of a ball-and-socket type $\mathcal{S}$ joint may be naturally characterized by a spherical cone representing the tilt motion of a direction vector and an unlimited spin (torsion) about it [3]. This suggests a tilt-torsion angle parameterization [4] (see Fig. 1(b), (c)) of the rotation generated by $\mathcal{S}$ joints. When the spin freedom is not required, a universal joint (denoted $\mathcal{U}$ ) is used instead. Due to physical limits, the tilt range of a ball-and-socket type $\mathcal{S}$ joint usually cannot exceed $45^{\circ}[2]$.

In order to overcome the limited tilt range of the $\mathcal{S}$ joint, alternative designs using kinematic chains have been proposed $[2,5,6]$. Closed loop spherical linkages have also been proposed $\mathcal{S}$ joints [7,8]. These designs are usually not compact enough for parallel machine tool design, and their tilt range is also quite small ${ }^{3}$.

In [10] a novel $2 \mathrm{R}$ parallel wrist with compact structure was proposed. The design is capable of tilting $90^{\circ}$, see Fig. 2 . When augmented by a revolute joint (denoted $\mathcal{R}$ ), it may serve as a novel, compact $\mathcal{S}$ joint with high stiffness and tilt range. This design was inspired by prior work on homokinetic joints [11-13]. A homokinetic or constant-velocity joint, which we denote by $\mathcal{O}$, provides the $2 \mathrm{R}$ freedoms for orientating a direction vector (say the $\mathbf{z}$-axis) without spinning. That is, having a zero torsion angle in the tilt-torsion angle parameterization.

${ }^{3}$ A reasonable benchmark would be $\pm 90^{\circ}$ tilt range along any tilt axis, which is required, for example, for five-face machining [9]. 


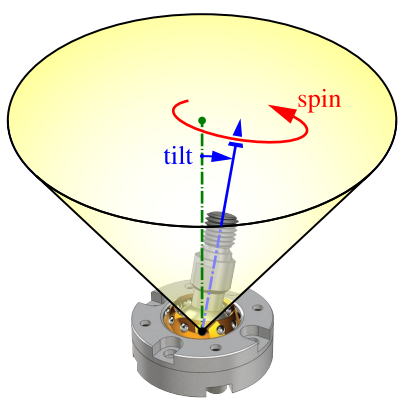

(a)

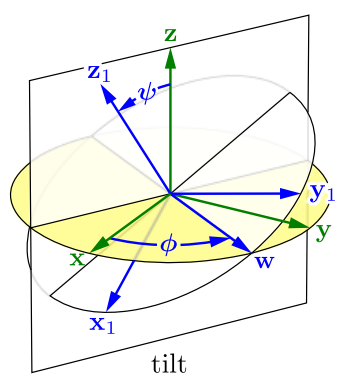

(b)

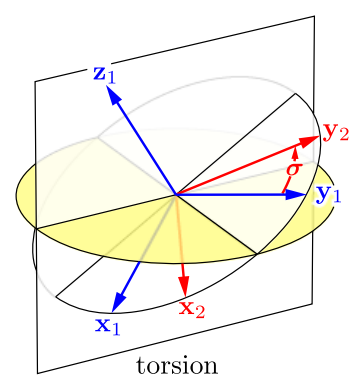

(c)

Fig. 1. (a) Workspace characteristics of a typical ball-and-socket spherical joint; (b) and (c) tilt-torsion angle parametrization.

The so-called zero-torsion PMs [14] may be considered as an $\mathcal{O}$ joint with additional translational freedoms. Closely related to our work is the plane-symmetric $\mathrm{N}-\mathcal{U U}$ PM [15] (rediscovered in [16,17]). This is a type of zero-torsion mechanism that does not fix the center of rotation, and therefore is not a spherical joint [18].

By comparison with existing design of $\mathcal{S}$ joints, our proposed $\mathcal{O}$ joint (augmented with a $\mathcal{R}$ joint if necessary) can be specifically designed to have a compact kinematic structure for increased stiffness while still retaining a large tilt range. This makes the $\mathcal{O}$ joint a novel machine element that could replace the commonly used $\mathcal{U}$ and $\mathcal{S}$ joints. A particularly successful application of the $\mathcal{O}$ joint, as we shall illustrate in this paper, is the synthesis of $1 \mathrm{~T} 2 \mathrm{R}$ zero-torsion PMs $[4,14,18]$.

\section{Basic Notations and Concepts}

The key to synthesizing an $\mathcal{O}$ joint and zero-torsion PMs is the underlying concept of a symmetric subspace and its associated Lie triple subsystem [19-22].

We represent a rigid-body motion $\mathbf{g} \in \mathrm{SE}(3)$ by a dual quaternion $\mathbf{q}_{0}+\varepsilon \mathbf{q}_{1}$ :

$$
\mathbf{q}_{0} \doteq a_{0}+a_{1} \mathbf{i}+a_{2} \mathbf{j}+a_{3} \mathbf{k}, \quad \mathbf{q}_{1} \doteq b_{0}+b_{1} \mathbf{i}+b_{2} \mathbf{j}+b_{3} \mathbf{k}
$$

where $\mathbf{i}, \mathbf{j}, \mathbf{k}$ denote the unit quaternions along $\mathbf{x}, \mathbf{y}$ and $\mathbf{z}$-axis, and $\varepsilon$ is the dual element satisfying $\varepsilon^{2}=0$. The coordinate $\left(a_{0}: a_{1}: a_{2}: a_{3}: b_{0}: b_{1}: b_{2}: b_{3}\right)$ of $\mathbf{g}$ satisfy the equation for the Study quadric $Q_{s}$ :

$$
Q_{s} \doteq\left\{\left(a_{0}: \cdots: b_{3}\right) \in \mathrm{P}^{7} \mid \sum_{i=0}^{3} a_{i} b_{i}=0\right\}
$$

in the seven-dimensional real projective space $\mathrm{P}^{7}$. The rigid displacement group $\mathrm{SE}(3)$ is identified with the non-ideal points of $Q_{s}$, namely points $\left(a_{0}: \cdots: b_{3}\right)$ satisfying $\sum_{i=0}^{3} a_{i}^{2} \neq 0$. Twists can be identified with dual vectors $\boldsymbol{\xi} \doteq \mathbf{w}+\varepsilon \mathbf{v}$, $\mathbf{w} \doteq w_{1} \mathbf{i}+w_{2} \mathbf{j}+w_{3} \mathbf{k}, \mathbf{v} \doteq v_{1} \mathbf{i}+v_{2} \mathbf{j}+v_{3} \mathbf{k}$. 


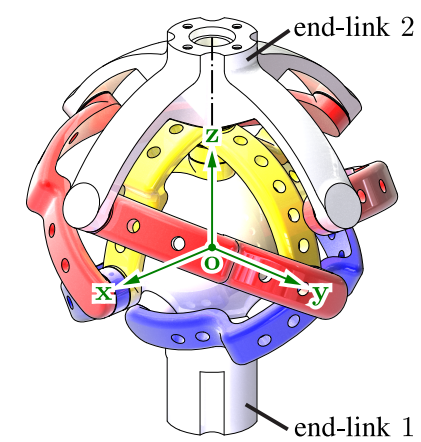

(a)

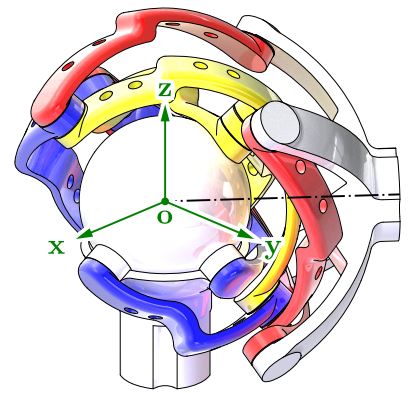

(b)

Fig. 2. Design implementation of a $\mathcal{O}$ joint [10]. (a) Home configuration; (b) tilted configuration.

The pitch of a twist $\boldsymbol{\xi}$, is given by $p \doteq(\mathbf{w} \cdot \mathbf{v}) /(\mathbf{w} \cdot \mathbf{w})$. A uniform screw motion about a constant twist $\boldsymbol{\xi}$ can be written as an exponential $\mathbf{g}=\exp \left(\frac{\theta}{2} \boldsymbol{\xi}\right)$, where $\theta$ parameterizes the motion. If the pitch is zero we have: $\exp \left(\frac{\theta}{2} \boldsymbol{\xi}\right)=$ $\left(\cos \frac{\theta}{2}+\sin \frac{\theta}{2} \boldsymbol{\xi}\right) \sim\left(1+\tan \frac{\theta}{2} \boldsymbol{\xi}\right)$, where $\sim$ denotes that the coordinates in the two expressions are equal up to a non-zero scalar. A pure translation parallel to a unit direction $\mathbf{v}$ is given by $1+\varepsilon \frac{t}{2} \mathbf{v}$, where $t$ is the distance moved. See $[23,24]$ for more details.

Consider an $\mathcal{O}$ joint located at the origin, with its space of infinitesimal twists coinciding with the xy-plane. It was shown in $[19,25]$ that the motion manifold of such a joint is a two-dimensional manifold given by,

$$
\begin{aligned}
\mathrm{M}_{2 B} & \doteq\left\{a_{0}+a_{1} \mathbf{i}+a_{2} \mathbf{j} \mid \sum_{i=0}^{2} a_{i}^{2} \neq 0\right\}=\exp (\operatorname{span}\{\mathbf{i}, \mathbf{j}\}) \\
& =\left\{\cos \frac{\psi}{2}+\sin \frac{\psi}{2} \mathbf{w} \mid \mathbf{w}=\cos \phi \mathbf{i}+\sin \phi \mathbf{j}, \phi \in[0,2 \pi), \psi \in[0, \pi]\right\}
\end{aligned}
$$

As a subvariety of $\mathrm{P}^{7}$ this is the 2-plane $a_{3}=b_{0}=b_{1}=b_{2}=b_{3}=0$, which lies inside $Q_{s}$; see [26]. As a manifold $\mathrm{M}_{2 B}$ is a symmetric subspace of $\mathrm{SO}(3)$ (and hence of $\mathrm{SE}(3))$ [19]. This has several implications for the synthesis of its motion generators ${ }^{4}$. Note that $\mathrm{M}_{2 B}$ is $\mathbf{z}$-axial-symmetric. That is, $\mathrm{M}_{2 B}$ is invariant under a conjugation by any rotation about the $\mathbf{z}$-axis. This is clear since,

$$
\begin{aligned}
\mathbf{q}_{0}\left(a_{0}+a_{1} \mathbf{i}+a_{2} \mathbf{j}\right) \mathbf{q}_{0}^{-1} & =\left(\cos \frac{\theta}{2}+\sin \frac{\theta}{2} \mathbf{k}\right)\left(a_{0}+a_{1} \mathbf{i}+a_{2} \mathbf{j}\right)\left(\cos \frac{\theta}{2}-\sin \frac{\theta}{2} \mathbf{k}\right) \\
& =a_{0}+\left(a_{1} \cos \theta-a_{2} \sin \theta\right) \mathbf{i}+\left(a_{1} \sin \theta+a_{2} \cos \theta\right) \mathbf{j} \in \mathrm{M}_{2 B}
\end{aligned}
$$

By contrast, the motion manifold of a $\mathcal{U}$ joint located at the origin and with its two axes aligned with the $\mathbf{x}$ and $\mathbf{y}$-axis:

$$
\mathbf{g} \sim\left(1+\tan \frac{\theta_{1}}{2} \mathbf{i}\right)\left(1+\tan \frac{\theta_{2}}{2} \mathbf{j}\right)=1+\tan \frac{\theta_{1}}{2} \mathbf{i}+\tan \frac{\theta_{2}}{2} \mathbf{j}+\tan \frac{\theta_{1}}{2} \tan \frac{\theta_{2}}{2} \mathbf{k}
$$

\footnotetext{
${ }^{4}$ Here, we have followed the same notation in [19]: a $m$-dimensional symmetric subspace of $\mathrm{SE}(3)$ with $1 \mathrm{R}$ and $2 \mathrm{R}$ DOFs is denoted by $\mathrm{M}_{m A}$ and $\mathrm{M}_{m B}$ respectively. The subscript $B$ is dropped for $m=4,5$.
} 
The motion manifold for the $\mathcal{U}$ joint is [27]:

$$
\mathrm{U} \doteq\left\{a_{0}+a_{1} \mathbf{i}+a_{2} \mathbf{j}+a_{3} \mathbf{k} \mid \sum_{i=0}^{3} a_{i}^{2} \neq 0, a_{0} a_{3}-a_{1} a_{2}=0\right\}
$$

As a subvariety of $\mathrm{P}^{7}$ this is the intersection of the Study quadric with a 3-plane. Unlike $\mathrm{M}_{2 B}$, this does not admit $\mathbf{z}$-axial-symmetry, showing that $\mathrm{M}_{2 B}$ cannot be generated by a $\mathcal{U}$ joint.

\section{Zero-torsion PM with Plane Symmetry}

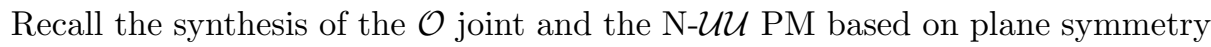
in [19], which allows us to use $\mathcal{O}$ joints as a basic machine element for the synthesis of zero-torsion PMs.

In [19], it was shown that $\mathrm{M}_{2 B}$ may be generated by an xy-plane-symmetric $\mathcal{R R} \mathcal{R}$ chain with convergent axes under symmetric movement condition, A $\mathcal{O}$ joint with four $\mathcal{R} \mathcal{R} \mathcal{R}$ chains was presented in [10]; see Fig. 2. According to Eq. (4), the $\mathcal{O}$ joint can be structurally synthesized with identical $\mathcal{R} \mathcal{R} \mathcal{R}$ legs laid out around the $\mathbf{z}$-axis with arbitrary angles (hereafter referred to as the phase angle for brevity) apart from each other. In practice, a phase angle of $90^{\circ}$ or $120^{\circ}$ is chosen for uniform kinetostatic behavior.

The 1T2R submanifold $\mathrm{M}_{3 B}$ [19], represented in dual quaternion as follows:

$$
\mathrm{M}_{3 B} \doteq\left\{a_{0}+a_{1} \mathbf{i}+a_{2} \mathbf{j}+\varepsilon b_{3} \mathbf{k} \mid \sum_{i=0}^{2} a_{i}^{2} \neq 0\right\}=\exp (\operatorname{span}(\mathbf{i}, \mathbf{j}, \varepsilon \mathbf{k}))
$$

is another instance of symmetric subspace of $\mathrm{SE}(3)$. It is the exponential image of the $B$-plane $\operatorname{span}(\mathbf{i}, \mathbf{j}, \varepsilon \mathbf{k})$ defined by linear equations $a_{3}=b_{0}=b_{1}=b_{2}=$ 0 , which is completely contained in the Study quadric $Q_{s}[26]$. Its mechanism synthesis can be shown to be governed by plane symmetry $[19,28]$. A kinematic chain generating $\mathrm{M}_{3 B}$ often has five freedoms, with the third joint lying in the $\mathbf{x y}$-plane and the first and the fifth (the second and the forth) being xy-planesymmetric. A total of three legs are needed to form a $\mathrm{M}_{3 B}$ PM [28]. Since $\mathrm{M}_{3 B}$ is conjugation invariant under planar displacements (i.e., transformation of the form $\left.a_{0}+a_{3} \mathbf{k}+\varepsilon\left(b_{1} \mathbf{i}+b_{2} \mathbf{j}\right)\right)$ [19], the legs of a $\mathrm{M}_{3 B}$ PM may be chosen so that each leg is conjugate to the other by an arbitrary planar motion.

Alternatively, with the $\mathcal{O}$ joint readily available as a basic machine element, we may form plane-symmetric chains with four freedoms as follows. Consider an $\mathcal{R O} \mathcal{R}$ chain with the two $\mathcal{R}$ joints being xy-plane-symmetric and the $\mathcal{O}$ joint situated in the xy-plane. For convenience, we assume the axes of the two $\mathcal{R}$ joints are both parallel to the $\mathbf{x}$-axis and pass through the points $-d \mathbf{z}$ and $d \mathbf{z}$ respectively; the $\mathcal{O}$ joint is located at a generic point $\mathbf{p}=\left(p_{x}, p_{y}, 0\right)^{T}$ on the xy-plane (see Fig. 3(a)).

The generic motion $\mathbf{h}$ of the $\mathcal{O}$ joint is given by:

$$
\begin{aligned}
\mathbf{h} & =\left(1+\varepsilon\left(\frac{p_{x}}{2} \mathbf{i}+\frac{p_{y}}{2} \mathbf{j}\right)\right)\left(a_{0}+a_{1} \mathbf{i}+a_{2} \mathbf{j}\right)\left(1-\varepsilon\left(\frac{p_{x}}{2} \mathbf{i}+\frac{p_{y}}{2} \mathbf{j}\right)\right) \\
& =\left(a_{0}+a_{1} \mathbf{i}+a_{2} \mathbf{j}\right)+\varepsilon\left(-p_{y} a_{1}+p_{x} a_{2}\right) \mathbf{k} \in \mathrm{M}_{3 B}
\end{aligned}
$$




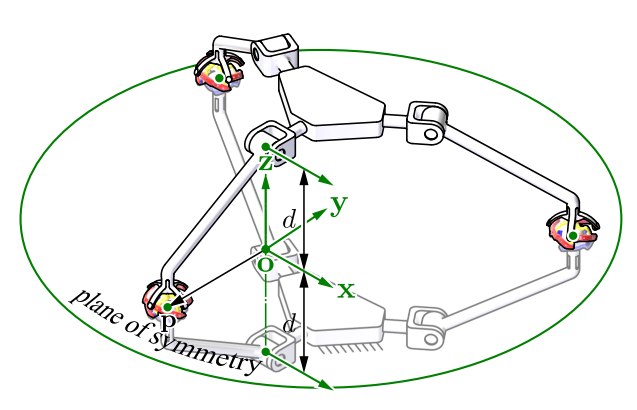

(a)

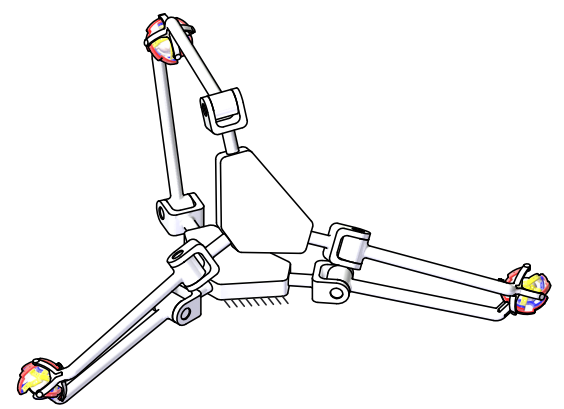

(b)

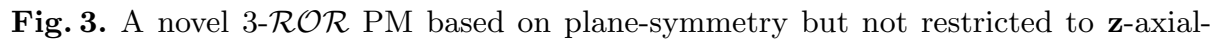
symmetry. (a) Home configuration; (b) tilted configuration.

A generic displacement $\mathrm{g}$ of the plane-symmetric $\mathcal{R O} \mathcal{R}$ chain under symmetric movement is then given by,

$$
\begin{aligned}
\mathbf{g}= & \left(1+\tan \frac{\theta}{2}(\mathbf{x}-\varepsilon d \mathbf{y})\right) \mathbf{h}\left(1+\tan \frac{\theta}{2}(\mathbf{x}+\varepsilon d \mathbf{y})\right) \\
\sim & \left(a_{0} \cos \theta-a_{1} \sin \theta\right)+\left(a_{1} \cos \theta+a_{0} \sin \theta\right) \mathbf{i}+a_{2} \mathbf{j}+\ldots \\
& \varepsilon\left(a_{0} d+a_{2} p_{x}-a_{1} p_{y}-a_{0} d \cos \theta+a_{1} d \sin \theta\right) \mathbf{k} \in \mathrm{M}_{3 B}
\end{aligned}
$$

showing that an $\mathcal{R O R}$ chain is a valid leg for a $\mathrm{M}_{3 B}$ PM. This leads to a novel $\mathrm{N}-\mathcal{R O} \mathcal{R}$ PM; see Fig. 3 for the case $\mathrm{N}=3$. A simple observation of its constraint wrench system shows that the $\mathrm{PM}$ is overconstrained for $\mathrm{N} \geq 2$. Notice that if we replace the $\mathcal{O}$ joints by $\mathcal{S}$ joints, we recover the $3-\mathcal{R S R}$ reflected tripod [28]. Similarly, we may also synthesize $\mathrm{M}_{3 B}$ PMs by replacing the plane-symmetric $\mathcal{R}$ joints in the $\mathcal{R O} \mathcal{R}$ legs with plane-symmetric $\mathcal{P}$ (prismatic) or $\mathcal{H}$ (helical) joints; see [19] for more details.

\section{1T2R Zero-torsion PM without Plane Symmetry}

Generally, we define a 1T2R zero-torsion motion manifold $M$ to be any threedimensional manifold that has the same twist space as $\mathrm{M}_{3 B}$ in its home configuration, and its $2 \mathrm{R}$ freedoms behaving as an $\mathcal{O}$ joint. The translational freedom is perpendicular to the plane of the instantaneous rotation axes in the home configuration, but it is generally not so away from it. A $1 \mathrm{~T} 2 \mathrm{R}$ zero-torsion PM can then be synthesized by legs with $2 \mathrm{R}$ freedoms (necessarily generated by a $\mathcal{O}$ joint) and one or more translational freedoms.

In the case of $1 \mathrm{~T} 2 \mathrm{R}$ legs, we only have the trivial case of a $\mathcal{P O}$ chain. By contrast, the motion manifold of any $3 \mathrm{~T} 2 \mathrm{R}$ leg is the five-dimensional symmetric subspace $\mathrm{M}_{5}$ [19]. In-parallel assemblies of 3T2R $\mathrm{M}_{5}$ legs with their characteristic directions [22] all parallel to each other necessarily produce a PM generating $\mathrm{M}_{5}$. Our investigation reduces to 4 -DOF legs of type $\mathcal{P} \mathcal{P O}$ or $\mathcal{P O P}$. In both cases, the direction vectors of the two $\mathcal{P}$ joints, say $\mathbf{v}_{1}$ and $\mathbf{v}_{2}$, span a plane 
perpendicular to the $\mathbf{x y}$-plane, and the $\mathcal{O}$ joint is located on the $\mathbf{x y}$-plane in the home configuration.

Consider, without loss of generality, a $\mathcal{P P O}$ chain with the $\mathcal{O}$ joint located at the origin, and with the $\mathbf{x z}$-plane as the $\mathbf{v}_{1} \mathbf{v}_{2}$-plane. A generic displacement $\mathrm{g}$ of the $\mathcal{P} \mathcal{P} \mathcal{O}$ chain is given by:

$$
\begin{aligned}
\mathbf{g} & =\left(1+\varepsilon\left(\frac{t_{1}}{2} \mathbf{i}+\frac{t_{2}}{2} \mathbf{k}\right)\right)\left(a_{0}+a_{1} \mathbf{i}+a_{2} \mathbf{j}\right) \\
& =\left(a_{0}+a_{1} \mathbf{i}+a_{2} \mathbf{j}\right)+\varepsilon \frac{1}{2}\left(-t_{1} a_{1}+\left(t_{1} a_{0}-t_{2} a_{2}\right) \mathbf{i}+t_{2} a_{1} \mathbf{j}+\left(t_{2} a_{0}+t_{1} a_{2}\right) \mathbf{k}\right)
\end{aligned}
$$

The coordinates of $\mathbf{g}$ can be shown to satisfy $a_{3}=0$, the Study quadric $Q_{s}$ and the following quadric:

$$
Q_{1}: a_{0} b_{2}-a_{1} b_{3}-a_{2} b_{0}=0
$$

When $\mathcal{O}$ is located at a generic point $\left(p_{x}, 0,0\right)$ and the $\mathbf{v}_{1} \mathbf{v}_{2}$-plane makes an angle $\alpha$ with the xz-plane, $Q_{s}$ and $a_{3}=0$ remain unchanged; $Q_{1}$ is given by (for conjugation operation on varieties, see $[27])$ :

$$
\begin{aligned}
Q_{1} & :\left(a_{0} b_{2}-a_{1} b_{3}-a_{2} b_{0}+a_{1} a_{2} p_{x}\right) \cos \alpha \ldots \\
& +\left(-a_{0} b_{1}+a_{1} b_{0}-a_{2} b_{3}+a_{2}^{2} p_{x}\right) \sin \alpha=0
\end{aligned}
$$

It is then clear that the displacements of a $2-\mathcal{P} \mathcal{P O}$ PM are, in general determined by the intersection of three quadrics and a hyperplane, namely the Study quadric $Q_{s}$, the two $Q_{1}$ 's for the two legs, and $a_{3}=0$. This gives rise to the desired 1T2R zero-torsion motion pattern.

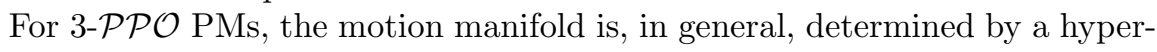
plane and four linearly independent quadrics and hence has only two freedoms. A general investigation of the 3 -DOF $\mathcal{P} \mathcal{P O}$ PM is beyond the scope of this paper. Instead, we consider the special case of a $\mathbf{z}$-axial-symmetric $3-\mathcal{P} \mathcal{P O}$ PM with identical $\mathcal{P} \mathcal{P O}$ legs, where the second and third leg have a phase angle of $120^{\circ}$ and $240^{\circ}$, respectively.

Applying the conjugation transformation to $Q_{1}$, we have the hyperplane $a_{3}=0$, the Study quadric $Q_{s}$, and the following quadrics constraining the displacements of the PM,

$$
\begin{aligned}
Q_{1}: & \left(a_{0} b_{2}-a_{1} b_{3}-a_{2} b_{0}+a_{1} a_{2} p_{x}\right) \cos \alpha \ldots \\
& +\left(-a_{0} b_{1}+a_{1} b_{0}-a_{2} b_{3}+a_{2}^{2} p_{x}\right) \sin \alpha=0 \\
Q_{1}^{\prime}: & \left(a_{0} b_{2}-a_{1} b_{3}-a_{2} b_{0}+a_{1} a_{2} p_{x}\right) \cos \left(\alpha+\frac{2 \pi}{3}\right) \ldots \\
& +\left(-a_{0} b_{1}+a_{1} b_{0}-a_{2} b_{3}+a_{2}^{2} p_{x}\right) \sin \left(\alpha+\frac{2 \pi}{3}\right) \ldots \\
& -2 p_{x} \sin \frac{\pi}{3}\left(a_{1} \cos \frac{\pi}{3}+a_{2} \sin \frac{\pi}{3}\right)\left(a_{1} \cos \left(\alpha+\frac{2 \pi}{3}\right)+a_{2} \sin \left(\alpha+\frac{2 \pi}{3}\right)\right)=0 \\
Q_{1}^{\prime \prime}: & \left(a_{0} b_{2}-a_{1} b_{3}-a_{2} b_{0}+a_{1} a_{2} p_{x}\right) \cos \left(\alpha+\frac{4 \pi}{3}\right) \ldots \\
& +\left(-a_{0} b_{1}+a_{1} b_{0}-a_{2} b_{3}+a_{2}^{2} p_{x}\right) \sin \left(\alpha+\frac{4 \pi}{3}\right) \ldots \\
& -2 p_{x} \sin \frac{2 \pi}{3}\left(a_{1} \cos \frac{2 \pi}{3}+a_{2} \sin \frac{2 \pi}{3}\right)\left(a_{1} \cos \left(\alpha+\frac{4 \pi}{3}\right)+a_{2} \sin \left(\alpha+\frac{4 \pi}{3}\right)\right)=0
\end{aligned}
$$

from which we have:

$$
Q_{1}+Q_{1}^{\prime}+Q_{1}^{\prime \prime}=\frac{3}{2}\left(a_{1}^{2}+a_{2}^{2}\right) p_{x} \sin \alpha
$$






(a)

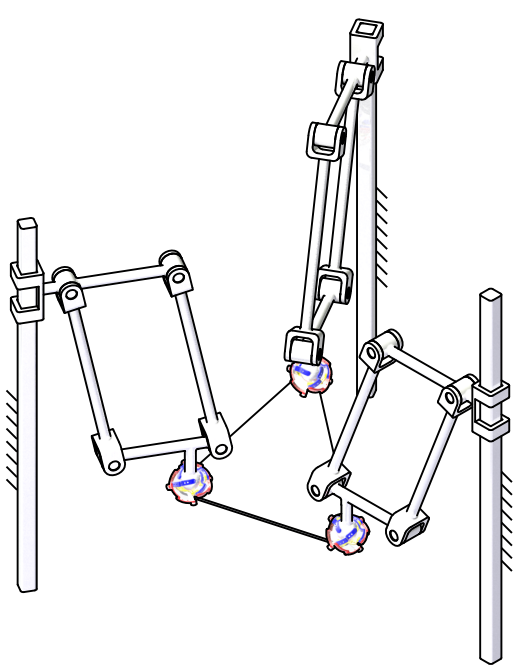

(b)

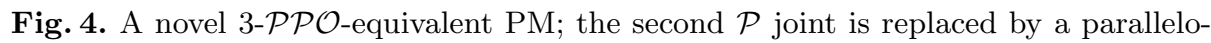
gram joint $\mathcal{P}_{a}$. (a) Home configuration; (b) tilted configuration.

This implies that the quadrics $Q_{1}, Q_{1}^{\prime}$ and $Q_{1}^{\prime \prime}$ become linearly dependent when $\alpha=0$, or when the $\mathbf{v}_{1} \mathbf{v}_{2}$-plane of the first leg is aligned with the $\mathbf{x z}$-plane, thus

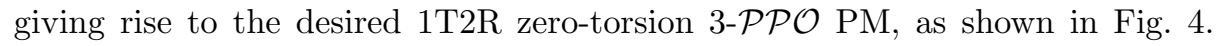
Substituting $\alpha=0$ into Eq. (13) gives the following two independent quadrics defining M:

$$
\begin{aligned}
& Q_{1}: a_{0} b_{2}-a_{1} b_{3}-a_{2} b_{0}+a_{1} a_{2} p_{x}=0 \\
& Q_{1}^{\prime}: a_{0} b_{1}-a_{1} b_{0}+a_{2} b_{3}-\frac{1}{2}\left(a_{1}^{2}-a_{2}^{2}\right) p_{x}=0
\end{aligned}
$$

Unlike $\mathrm{M}_{2 B}$ or $\mathrm{M}_{3 B}$, $\mathrm{M}$ does not admit a continuous $\mathrm{z}$-axial-symmetry, but instead admits a discrete three-fold symmetry. Notice that when the $\mathcal{O}$ joints

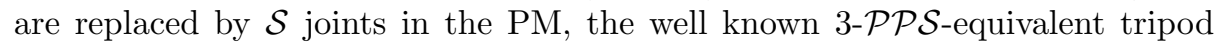
mechanism is recovered [14].

As in the previous subsection, a 2-POP PM is, in general, guaranteed to have the desired 1T2R zero-torsion motion manifold M. We investigate the following 3-POP PM with a special-geometry given by locating the $\mathcal{O}$ joint of the first leg at $\left(p_{x}, 0,0\right)$; and the direction vector for the first and the third $\mathcal{P}$ joint placed at $(\sin \beta, 0, \cos \beta)$ and $(\cos \gamma, 0, \sin \gamma)$, respectively. The PM is three-fold $\mathbf{z}$-axialsymmetric. A generic displacement $\mathbf{g}$ of the first leg is given by,

$$
\begin{aligned}
\mathbf{g}=\left(1+\varepsilon\left(\frac{t_{1}}{2} \sin \beta \mathbf{i}+\frac{t_{1}}{2} \cos \beta \mathbf{k}\right)\right) \ldots \\
\quad\left(a_{0}+a_{1} \mathbf{i}+a_{2} \mathbf{j}+\varepsilon p_{x} a_{2} \mathbf{k}\right)\left(1+\varepsilon\left(\frac{t_{2}}{2} \cos \gamma \mathbf{i}+\frac{t_{2}}{2} \sin \gamma \mathbf{k}\right)\right)
\end{aligned}
$$




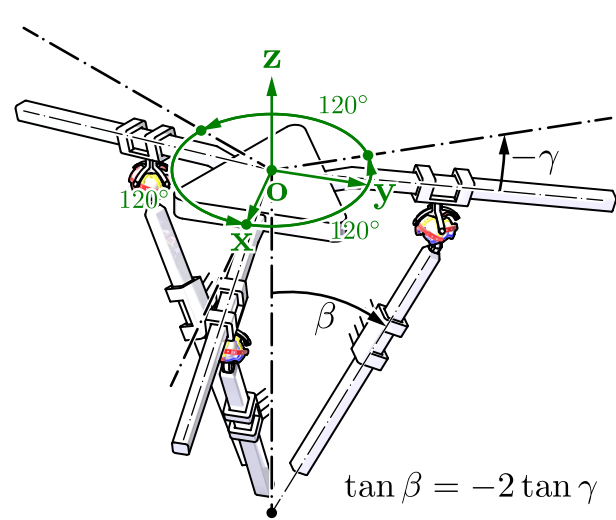

(a)

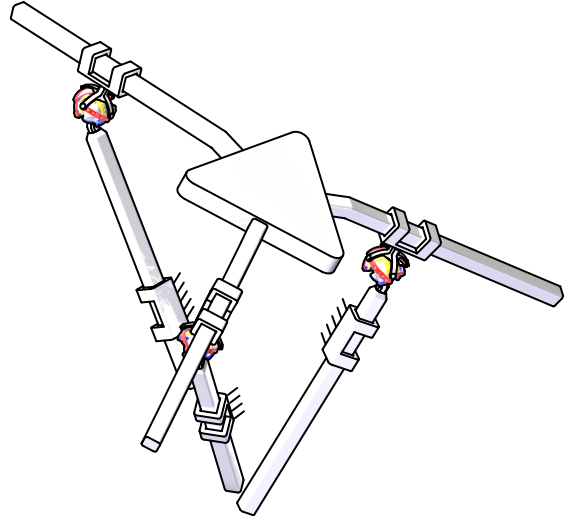

(b)

Fig. 5. A novel 3-POOP PM under the condition $\tan \beta=-2 \tan \gamma$. (a) Home configuration; (b) tilted configuration.

which satisfies $a_{3}=0$, the Study quadric $Q_{s}$, and the following quadric:

$$
\begin{aligned}
& Q_{2}: 2 \cos \beta \sin \gamma a_{0} b_{0}-\cos (\beta+\gamma) a_{0} b_{2} \ldots \\
& \quad+\cos (\beta-\gamma) a_{1} b_{3}-\cos (\beta+\gamma) a_{2} b_{0}-2 \cos \gamma \sin \beta a_{2} b_{2}=0
\end{aligned}
$$

As in Eq. (14), after summing the second quadrics of the three legs we have,

$$
Q_{2}+Q_{2}^{\prime}+Q_{2}^{\prime \prime}=3 a_{0} b_{0}(\cos \gamma \sin \beta+2 \cos \beta \sin \gamma)
$$

When $\tan \beta=-2 \tan \gamma$, the three quadrics become linearly dependent and give rise to a $1 \mathrm{~T} 2 \mathrm{R}$ zero-torsion $3-\mathcal{P O P}$ PM (as shown in Fig. 5), with the corresponding motion manifold $\mathrm{M}$ defined by $a_{3}=0$, the Study quadric $Q_{s}$, and the following quadrics:

$$
\begin{aligned}
Q_{2}: \quad \tan \beta a_{0} b_{0}+\left(1+\frac{1}{2} \tan \beta^{2}\right) a_{0} b_{2}-\left(1-\frac{1}{2} \tan \beta^{2}\right) a_{1} b_{3} \ldots \\
\quad+\left(1+\frac{1}{2} \tan \beta^{2}\right) a_{2} b_{0}+2 \tan \beta a_{2} b_{2}=0 \\
Q_{2}^{\prime}: \tan \beta a_{0} b_{0}-\frac{\sqrt{3}}{2}\left(1+\frac{1}{2} \tan \beta^{2}\right) a_{0} b_{1}-\frac{1}{2}\left(1+\frac{1}{2} \tan \beta^{2}\right) a_{0} b_{2} \ldots \\
\quad-\frac{\sqrt{3}}{2}\left(1+\frac{1}{2} \tan \beta^{2}\right) a_{1} b_{0}+\frac{3}{2} \tan \beta a_{1} b_{1}+\frac{\sqrt{3}}{2} \tan \beta a_{1} b_{2} \ldots \\
\quad+\frac{1}{2}\left(1-\frac{1}{2} \tan \beta^{2}\right) a_{1} b_{3}-\frac{1}{2}\left(1+\frac{1}{2} \tan \beta^{2}\right) a_{2} b_{0} \ldots \\
\quad+\frac{\sqrt{3}}{2} \tan \beta a_{2} b_{1}+\frac{1}{2} \tan \beta a_{2} b_{2}-\frac{\sqrt{3}}{2}\left(1-\frac{1}{2} \tan \beta^{2}\right) a_{2} b_{3}=0
\end{aligned}
$$

Unlike $\mathrm{M}_{2 B}$ or $\mathrm{M}_{3 B}$, $\mathrm{M}$ in general does not admit a continuous z-axial-symmetry, but instead admits a discrete three-fold symmetry.

Finally, notice that when the $\mathcal{O}$ joints are replaced by $\mathcal{S}$ joints in the PM, we recover the $3-\mathcal{P S P} P M$ [14]. It is interesting to note that, in the case of 
$\beta=\gamma=0$, Eq. (19) reduces to:

$$
\begin{aligned}
& Q_{2}: a_{0} b_{2}-a_{1} b_{3}+a_{2} b_{0}=0 \\
& Q_{2}^{\prime}: a_{0} b_{1}+a_{1} b_{0}+a_{2} b_{3}=0
\end{aligned}
$$

which, along with $a_{3}=0$ and the Study quadric $Q_{s}$, defines a $\mathbf{z}$-axial-symmetric motion manifold M. This implies, surprisingly, that the particular $3-\mathcal{P S} \mathcal{P}$ PM studied in [14] can indeed have arbitrary leg phase angle, and in particular does not have to be three-fold $\mathbf{z}$-axial-symmetric.


$\mathcal{P U} \mathcal{P}$ PM built in a similar fashion [29], but exhibits partitioned $1 \mathrm{~T} 2 \mathrm{R}$ freedoms.

\section{Conclusion}

This paper proposed a novel class of $1 \mathrm{~T} 2 \mathrm{R}$ zero-torsion PMs using homokinetic joints as basic machine elements. By representing the motion manifold using dual quaternions, we were able to investigate the axial symmetry properties of these spaces and derive synthesis conditions for their existence.

\section{References}

1. J.-P. Merlet, Parallel robots. Springer Science \& Business Media, 2006, vol. 128.

2. P. Pham, "Design of hybrid-kinematic mechanisms for machine tools," Ph.D. dissertation, EPFL, 2009.

3. R. P. Paul and C. N. Stevenson, "Kinematics of robot wrists," The International journal of robotics research, vol. 2, no. 1, pp. 31-38, 1983.

4. I. A. Bonev and J. Ryu, "A new approach to orientation workspace analysis of 6-DOF parallel manipulators," Mechanism and machine theory, vol. 36, no. 1, pp. 15-28, 2001.

5. L. F. Bieg and G. L. Benavides, "Large displacement spherical joint," U.S. Patent 6,409,413, Jun. 25, 2002.

6. L.-T. Schreiber and C. Gosselin, "Passively driven redundant spherical joint with very large range of motion," Journal of Mechanisms and Robotics, vol. 9, no. 3, p. $031014,2017$.

7. G. J. Hamlin and A. C. Sanderson, Tetrobot: A modular approach to reconfigurable parallel robotics. Springer, 1998.

8. P. Bosscher and I. Ebert-Uphoff, "A novel mechanism for implementing multiple collocated spherical joints," in Robotics and Automation, 2003. Proceedings. ICRA'03. IEEE International Conference on, vol. 1. IEEE, 2003, pp. 336-341.

9. J. Kim, F. Park, and J. Lee, "A new parallel mechanism machine tool capable of five-face machining," CIRP Annals-Manufacturing Technology, vol. 48, no. 1, pp. 337-340, 1999.

10. Y. Wu and M. Carricato, "Design of a novel 3-DoF serial-parallel robotic wrist: A symmetric space approach," in Proceedings of the International Symposium on Robotics Research (ISRR 2015), Sestri Levante, Italy. Springer, 2015.

11. M. Carricato, "Decoupled and homokinetic transmission of rotational motion via constant-velocity joints in closed-chain orientational manipulators," Journal of Mechanisms and Robotics, vol. 1, no. 4, p. 041008, 2009. 
12. G. A. Thompson, "Constant velocity coupling and control system therefor," U.S. Patent 7,144,326, Dec. 5, 2006.

13. H. Kocabas, "Design and analysis of a spherical constant velocity coupling mechanism," Journal of Mechanical Design, vol. 129, no. 9, pp. 991-998, 2007.

14. I. Bonev, D. Zlatanov, and C. Gosselin, "Advantages of the modified euler angles in the design and control of pkms," in 2002 Parallel Kinematic Machines International Conference, 2002, pp. 171-188.

15. I. H. Culver, "Constant velocity universal joint," U.S. Patent 3,477,249, Nov. 11, 1969.

16. M. E. Rosheim and G. F. Sauter, "New high-angulation omni-directional sensor mount," in Proceedings of SPIE, vol. 4821, 2002, pp. 163-174.

17. K. Sone, H. Isobe, and K. Yamada, "High angle active link," Special Issue Special Supplement to Industrial Machines, 2004.

18. Y. Wu, Z. Li, and J. Shi, "Geometric properties of zero-torsion parallel kinematics machines," in Intelligent Robots and Systems (IROS), 2010 IEEE/RSJ International Conference on. IEEE, 2010, pp. 2307-2312.

19. Y. Wu, H. Löwe, M. Carricato, and Z. Li, "Inversion symmetry of the euclidean group: Theory and application to robot kinematics," IEEE Transactions on Robotics, vol. 32, no. 2, pp. 312-326, 2016.

20. Y. Wu and M. Carricato, "Identification and geometric characterization of lie triple screw systems and their exponential images," Mechanism and Machine Theory, vol. 107, pp. 305-323, 2017.

21. J. Selig, "A class of explicitly solvable vehicle motion problems," IEEE Transactions on Robotics, vol. 31, no. 3, pp. 766-777, 2015.

22. Y. Wu and M. Carricato, "Symmetric subspace motion generators," IEEE Transactions on Robotics, vol. 34, no. 3, pp. 716-735, June 2018.

23. J. M. Selig, Geometric fundamentals of robotics. Springer, 2004.

24. J. M. McCarthy and G. S. Soh, Geometric design of linkages. Springer, 2006, vol. 11, 2ed.

25. Y. Wu, A. Müller, and M. Carricato, "The 2D orientation interpolation problem: a symmetric space approach," in Advances in Robot Kinematics. Springer, 2016.

26. J. Selig, Y. Wu, and M. Carricato, "Motion interpolation in lie subgroups and symmetric subspaces," in Computational Kinematics. Springer, 2018, pp. 467474.

27. J. M. Selig, "Displacement varieties for some pup linkages," in International Symposium on Advances in Robot Kinematics. Springer, 2018, pp. 28-36.

28. K. Hunt, "Constant-velocity shaft couplings: a general theory," Journal of Engineering for Industry, vol. 95, no. 2, pp. 455-464, 1973.

29. K. Zhang, J. S. Dai, and Y. Fang, "Constraint analysis and bifurcated motion of the 3pup parallel mechanism," Mechanism and Machine Theory, vol. 49, pp. 256-269, 2012. 